\title{
Effects of coenzyme Q10 on health-related quality of life, clinical disease activity and blood pressure in patients with mild to moderate ulcerative colitis: a randomized clinical trial
}

\author{
Farnaz Farsi ${ }^{1}$, Nasser Ebrahimi-Daryani ${ }^{2}$, Mahmood Barati ${ }^{3,4}$, Leila Janani ${ }^{5}$, Mohammad Yahya Karimi ${ }^{6}$, Abolfazl Akbari ${ }^{7}$,

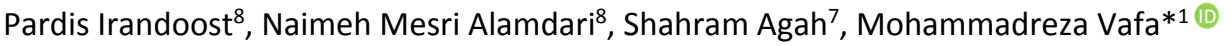

Received: 9 Apr 2020

Published: 6 Jan 2021

\section{Abstract}

Background: Ulcerative colitis (UC) is specified by a chronic mucosal inflammation that has a deleterious impact on the quality of life (QoL). Coenzyme Q10 (CoQ10) appears to influence disease activity by its obvious properties. Therefore, the current research intends to assess the impacts of CoQ10 on QoL, disease activity, and blood pressure in UC patients.

Methods: This clinical trial performed on men and women with UC in 2017 who were attended the gastrointestinal center of Hazrat Rasool Akram Hospital and private clinic. Eighty-eight UC patients were randomly allocated to receive either CoQ10 (200 mg/day) or placebo for 8 weeks. The anthropometric parameters, blood pressure, inflammatory bowel disease questionnaire-32 (IBDQ-32) score, and the Simple Clinical Colitis Activity Index (SCCAI) score were measured pre and post-intervention. P-value $<0.05$ was considered to be statistically significant. All statistical analysis was done using SPSS software version 24.

Results: Eighty-six UC patients (44 males) with a mean age of 39.29 (10.19) years completed the trial. The results of between- and within-group analysis revealed that the SCCAI score $(p<0.001$ and $p<0.001$, respectively), diastolic blood pressure ( $p=0.025$ and $\mathrm{p}=0.001$, respectively), and systolic blood pressure $(\mathrm{p}=0.001$ and $\mathrm{p}<0.001$, respectively) decremented significantly; while, the mean IBDQ-32 ( $<<0.001$ and $p=0.001$, respectively) increased substantially in the CoQ10 group; whereas there was no significant difference in anthropometric indices in both groups.

Conclusion: Findings suggest that CoQ10 can be used as a potential intervention for diminishing the disease severity and blood pressure and may improve QoL and UC patients.

IRCT number: IRCT20090822002365N17.

Keywords: Coenzyme Q10, Quality of life, Ulcerative colitis, SCCAI score, IBDQ-32

\author{
Conflicts of Interest: None declared \\ Funding: None \\ *This work has been published under CC BY-NC-SA 1.0 license. \\ Copyright $\odot$ Iran University of Medical Sciences
}

Cite this article as: Farsi F, Ebrahimi-Daryani N, Barati M, Janani L, Karimi MY, Akbari A, Irandoost P, Mesri Alamdari N, Agah Sh, Vafa M. Effects of coenzyme Q10 on health-related quality of life, clinical disease activity and blood pressure in patients with mild to moderate ulcerative colitis: a randomized clinical trial. Med J Islam Repub Iran. 2021 (6 Jan);35:3. https://doi.org/10.47176/mjiri.35.3

Corresponding author: Dr Mohammadreza Vafa, vafa.m@iums.ac.ir

1. Department of Nutrition, School of Public Health, Iran University of Medical Sciences, Tehran, Iran

2. Division of Gastroenterology, Imam Khomeini Hospital, School of Medicine, Tehran University of Medical Sciences, Tehran, Iran

3. Cellular and Molecular Research Center, Iran University of Medical Sciences, Tehran, Iran

4. Department of Medical Biotechnology, Faculty of Allied Medicine, Iran University of Medical Science, Tehran, Iran

5. Preventive Medicine and Public Health Research Center, Psychosocial Health Research Institute (PHRI), Iran University of Medical sciences, Tehran, Iran

6. Razi Drug Research Center, Iran University of Medical Sciences, Tehran, Iran

7. Colorectal Research Center, Iran University of Medical Sciences, Tehran, Iran

8. Student Research Committee, Department of Nutrition, School of Public Health, Iran University of Medical sciences, Tehran, Iran $\uparrow$ What is "already known" in this topic:

Coenzyme Q10 (CoQ10) is regarded as a well-known intracellular antioxidant that may reduce inflammation and oxidative stress in favor of the therapeutic efficacy of ulcerative colitis (UC). UC is a type of inflammatory condition of the colon that has detrimental impacts on quality of life.

\section{$\rightarrow$ What this article adds:}

Numerous evidence exists that the implications of CoQ10 supplementation in human diseases are related to chronic inflammation. CoQ10 seems chiefly to purpose the inflammatory cascade as a mechanism to diminish continuous disease activity. Therefore, it may be a better choice compared to current treatments in patients with UC. 


\section{Introduction}

Ulcerative colitis (UC) represents a chronic inflammatory digestive tract illness characterized by recurring flares followed by alternating periods of remission and relapses restricted to the rectal, colonic mucosal and submucosal layers (1). This disorder belongs to the initial subtype of Inflammatory Bowel Disease (IBD) of hitherto unknown origins and uncertain pathogenesis $(2,3)$. Population-based studies indicated that the incidence and prevalence of IBD are rising rapidly in low- and middle-income countries that have become a global disease in the 21 st century $(4,5)$. A recent nationwide report in Iran showed that the national incidence and prevalence of IBD and its burden appears to have similar trends $(6,7)$. The majority of patients suffer from intestinal complications of UC, such as cramping, diarrhea, hematochezia, and urgency to defecate, as well as experience adverse treatment effects $(3,8)$. UC, as a disabling disease, can adversely affect all domains of daily patient's life due to its chronic character, UC- related symptoms, young age of onset and medical treatment (9). Consequently, this disorder leads considerably to impairment and the reduction of Health-Related Quality of Life (HRQOL) of the individuals in many ways as well as imposing a substantial social and economic burden on the sufferer $(3,10,11)$. Similarly, HRQOL in IBD patients can also be influenced by sociodemographic factors such as gender, smoking, education level, work disability and unemployment (9). Previous studies have also shown that disease activity was considered as the strongest predictor of HRQOL in UC patients $(3,12,13)$. While the severity of IBD was assessed by using activity indexes, this conventional way was unable to consider other aspects that may affect the patient's life (10). Hence, obtaining appropriate information about the symptoms and patient characteristics related to poor HRQOL has a recognized importance for identifying patients needing special attention (14). As a result, HRQOL, a strong, independent predictor of health outcomes in clinical researches is applied to appraise the disease consequences in the daily life of an individual (15). The most widely used and validated disease-specific HRQOL instrument in IBD is the 32-item Inflammatory Bowel Disease Questionnaire (IBDQ), which enables the investigation of the four domains of bowel, systemic, emotional and social functioning, as well as the severity of the disease $(14,16-18)$. Hence, it assumes that improving the life quality of UC patients and its reaching to the levels of healthy individual's quality of life can be considered as one of the main goals therapeutic of UC (3). In this regard, it has been confirmed based on previous reports that total antioxidant intake has an association with recovery in HRQOL and severity of clinical disease $(15,19)$. Given that rising pro-inflammatory and/or decreasing anti-inflammatory cytokines are involved in UC development which is the cornerstone of treatment (8). Among of exogenous dietary factors, Coenzyme Q10 (CoQ10) in light of potent antioxidant property and its role in the mitochondrial energy generation appears to have beneficial effects on aspects of HRQOL, such as fatigue and pain $(15,20,21)$. In addition, the coloprotective effect of CoQ10 in experimental models of UC has been recently observed $(22,23)$. CoQ10 (also known as ubiquinone) is a vitamin-like substance with multi-functions, naturally synthesized by the body and can be obtained through the diet or orally as a dietary supplement $(24,25)$. However, its synthesis may diminish due to the lack of nutrients involving in CoQ10 synthesis, inflammation and oxidative stress circumstances of some chronic disease that lead to increasing the tissue's requirement for CoQ10 (16). Several investigations have been performed to assess the CoQ10 efficacy on HRQOL in patients of clinical trials $(15,20,21,24,26-28)$; In some of them, the link between CoQ10 status and HRQOL in the Iranian population was seen $(15,29)$. To our knowledge, no clinical studies have yet been carried out regarding the impact of CoQ10 supplementation in patients with UC around the world. Therefore, the present research purposes to determine the effectiveness of CoQ10 on the quality of life, clinical disease activity and blood pressure in patients with UC.

\section{Methods}

\section{Study Design}

This current project is the first report of a double-blind, randomized controlled trial with a parallel design trial and 1:1 ratio of two groups. Those patients who were attended to the Gastroenterology center at Rasoul-e-Akram Hospital and private clinic in Tehran were invited to participate in the study and assess underlying inclusion criteria during the investigation period (from April 2018 to March 2019).

\section{Ethical Considerations}

At the first visit, the investigator who was blinded fully described the project objectives, method of intervention, and the study period. Furthermore, it was explained that all participants would either be in the intervention group or in the placebo group. Afterward, a written informed consent form was signed by all patients before the intervention. The trial protocol was confirmed by the medical ethics committee of Iran University of Medical Sciences (IUMS), Tehran, Iran with certificate No: IR.IUMS.REC 1396.31412. Besides, the research has been registered at the Iranian Registry of Clinical Trials (IRCT): IRCT20090822002365N17.

\section{Inclusion and exclusion criteria}

Diagnosis of UC in research participants was previously confirmed base on the conventional clinical, colonoscopy, radiologic, and histological criteria, and their disease statuses were in a mild to moderate remission. Additional eligibility criteria were as follows: (1) their tendency to take part in the study; (2) a history of UC more than 6 months (3) men and women older than 18 years (4) body mass index (BMI) higher than 18.5 or lower than $30 \mathrm{~kg} / \mathrm{m}^{2}$. While the exclusion criteria were as follows: current alcohol and/or drug abuse; an inability to complete the questionnaire, taking of anti-coagulation drugs (heparin and warfarin), corticosteroids, antihistamines, calcium channel antagonists, any antioxidant supplements such as (vitamin $\mathrm{C}$, E, and omega 3), and lipid-modifying drugs 6 months prior to the study; existence of documented mental disorders; current treatment anti-TNF-alpha agent; patients with Clostridium difficile stool culture-positive samples; history of 
other intestinal disorders, cancer, inflammatory diseases, and autoimmune diseases; pregnancy and breastfeeding; the recurrence and flare-up of the disease that needed hospitalization; and altering the treatment protocols during the intervention.

\section{Randomization and intervention}

Eligible participants were randomized by using the permuted blocked randomization method with quadruple blocks. Given the sample size of 88 identified, 22 blocks were generated through the online site (www.sealedenvelope.com); besides, the allocation concealment process was performed by a blinded statistician, who provided unique sequential codes by the software. In order to keep the investigators and participants uninformed about the intervention type of each group, the pharmaceutical boxes were assigned each individual into a study based on the sequence produced by one staff of the Gastroenterology clinic, who was also blind. Subsequently, for all the bottles containing capsules. Subsequently, the code of each bottle assigned to the patient was registered. Each patient in the intervention and control groups, in addition to regular medication, either took a capsule of CoQ10 $100 \mathrm{mg}$ (produced by the Nutri Century Company, Canada) and placebo orally twice daily, respectively. All the participants were followed for 8 weeks. Placebo capsules contained rice flour similar in appearance and color to the CoQ10 capsules.

The choice of dose and duration of the CoQ10 administration has been arbitrarily selected owing to the lack of previous human experience. Besides investigators throughout the enrollment and data collection processes and participants were kept blind. All participants were instructed to avoid taking any additional supplements containing CoQ10 or antioxidants during the trial. Adherence to study intervention was investigated by counting the leftover capsules at the end of the trial. If $90 \%$ of capsules were taken from the patients studied, the compliance with them was considered.

\section{Measurements}

At baseline and 8-week follow-up, anthropometric characteristics were as follows: the height of participants was recorded without shoes, to $0.5-\mathrm{cm}$ accuracy using a Seca stadiometer. The participant's weight was measured using a 0.1-kg accuracy on a digital scale (Beurer Co., Germany) with wearing light clothes and no shoes. The patient's BMI was computed as the ratio of an individual's weight in kilograms divided by the height in meters squared. Using nonstretchable tape, without any pressure applied to the surface of the body, the measurement of waist circumference (WC) (distance between the lowest ribs to the iliac spine) and hip circumference (HC) (the greatest circumference size of the hip) were performed. These measurements were recorded with a $0.1-\mathrm{cm}$ accuracy. The Waist-to-hip ratio (WHR) is obtained by dividing the WC by the HC. It should also be mentioned that the anthropometric measurements following overnight fasting for each participant were done by the researcher exactly before blood sampling at the lab, which was in the morning before at 9.00 o'clock at baseline and endpoint.
Participant's blood pressure also measured by digital monitoring after at least five minutes of resting in the sitting position at the beginning and the end of the study.

At the onset of this RCT, a general demographics questionnaire (age, sex, medicine, clinical data, history smoking) for each patient was completed using a self-administered. To evaluate patients' diets, 24-hour food recalls (one ordinary day and one weekend day) were also collected from all participants at baseline and endpoint. Data from a 2-day food record were converted to calorie and nutrient intakes and finally, analyze using a Nutritionist IV software, modified for Iranian foods (version 3.5.2, First Data Bank; Hearst Corp, San Bruno, CA).

\section{Evaluation of disease activity}

The clinical status of UC patients was determined using the Simple Clinical Colitis Activity Index Questionnaire (SCCAIQ) at pre- and post-intervention stages $(8,30)$. The SCCAI comprises a frequency of defecation during the day and night, the urgency of defecation, rectal bleeding, a physician's assessment of disease severity, and extraintestinal manifestations, which classifies patients into three grades of disease severity: inactive UC (i.e., in remission) and active UC (i.e., mild, moderate, and severe). Its total score can be from 0 to above 16 .

\section{Assessment of HRQOL}

The HRQOL status was determined using the IBDQ of the 32 multiple-choice self-report questions (18). This disease-specific questionnaire is grouped into four health subscales: bowel symptoms (10 questions); systemic symptoms, including sleep disorders and fatigue (5 questions); emotional function such as depression, aggression, and irritation (12 questions); and social function, meaning the ability to participate in social activities and to work (5 questions). Consequently, its total score ranges from 32 to 224 points, with lower scores reflecting worse HRQOL. Responses for each question are scored using a 7-point Likert scale ranging from 1 (low QOL) to 7 (high QOL), in which corresponding 7 to the best level of functioning. The IBDQ has been documented to be a reliable and sensitive tool to measure HRQOL (18). All patients were asked to complete IBDQ-32 questionnaires and to choose one of seven graded responses of HRQOL at the beginning and the end of the study duration.

\section{Measurements of physical activity}

We used the International Physical Activity Questionnaires (IPAQ) with a face-to-face interview format to examine the physical activity level of the patients during the last 7 days (31). Indeed, energy consumption was calculated based on the second edition of codes and metabolic equivalent (MET) values. The IPAQ data were converted to MET scores (MET-min/week) for each type of activity by multiplying the number of minutes. Furthermore, based on the revised scoring protocol 2005, physical activity levels were classified into 3 levels as follows: vigorous (at least $3000 \mathrm{MET}-\mathrm{min} /$ week), moderate (at least $600 \mathrm{MET}$ $\mathrm{min} / \mathrm{week}$, and low (less than $600 \mathrm{MET}-\mathrm{min} / \mathrm{week}$ ). This additional indicator variable is defined as the time spent in 
a sedentary position and is not comprised as a score of physical activity. This variable was calculated based on a minute per day. Its validity and reliability have been endorsed by many reports to survey physical activity (32).

\section{Statistical Analysis}

At first, the normality of each variable prior to data analysis was investigated using the graphical methods, numerical characteristics and Shapiro-Wilk's tests. Continuous quantitative variables were described as mean (standard deviation) or median $\left(25^{\text {th }}, 75^{\text {th }}\right)$ for the description of the central tendency and dispersion. The mean of quantitative variables between the two groups was compared by independent-sample t-test; while, if the distribution of data was not normal, Mann-Whitney U-test (as a non-parametric test) was used which had been displayed to be significantly different between groups at the baseline of the study; In addition, within-group analysis for the evaluation of changes induced by CoQ10 treatment were done using paired t-test and Wilcoxon's signed-rank in normally and non-normally distributed data, respectively. A chi-square test or Fisher's exact test was also performed to compare the categorical variables between the two randomized groups, and statistics were presented as frequency (percentage). Adjusted between-groups comparisons were done using parametric and non-parametric ANCOVA considering baseline values of outcomes as covariates, which include anthropometric measurements, SCCAIQ, IBDQ-32, SBP, DBP, and IPAQ. Between-groups comparisons considering the baseline value of the outcome, treatment type, carbohydrate, iron and fiber as covariates were done using linear regression, and bootstrap p-values were reported regarding the nonnormal distribution of outcomes. The statistical analysis of data without awareness of the group in which received treatment was carried out by using SPSS software version 24 (IBM Corp. IBM SPSS Statistics for Windows, Armonk, NY). Non-parametric ANCOVA analysis was done with R software (package "sm" in R-3.5.1 for windows). P-values less than 0.05 were regarded to be statistically significant. Modified ITT approach was used for the analysis of data because of removing two patients due to the medical protocol change during the 8-week follow-up.

\section{Results}

\section{Baseline characteristics}

In total, 177 patients with a definite diagnosis of UC by gastroenterologist were screened in this trial. Among them, 89 were excluded because of ineligible inclusion criteria and SCCAI score. Among the 88 patients with mild to moderate UC enrolled and randomized, 44 patients received a CoQ10 supplement, and 44 received a placebo in addition to the optimized oral and topical drugs in all patients. Two patients in CoQ10 and placebo arms were excluded from the study due to the medical protocol change during the 8-

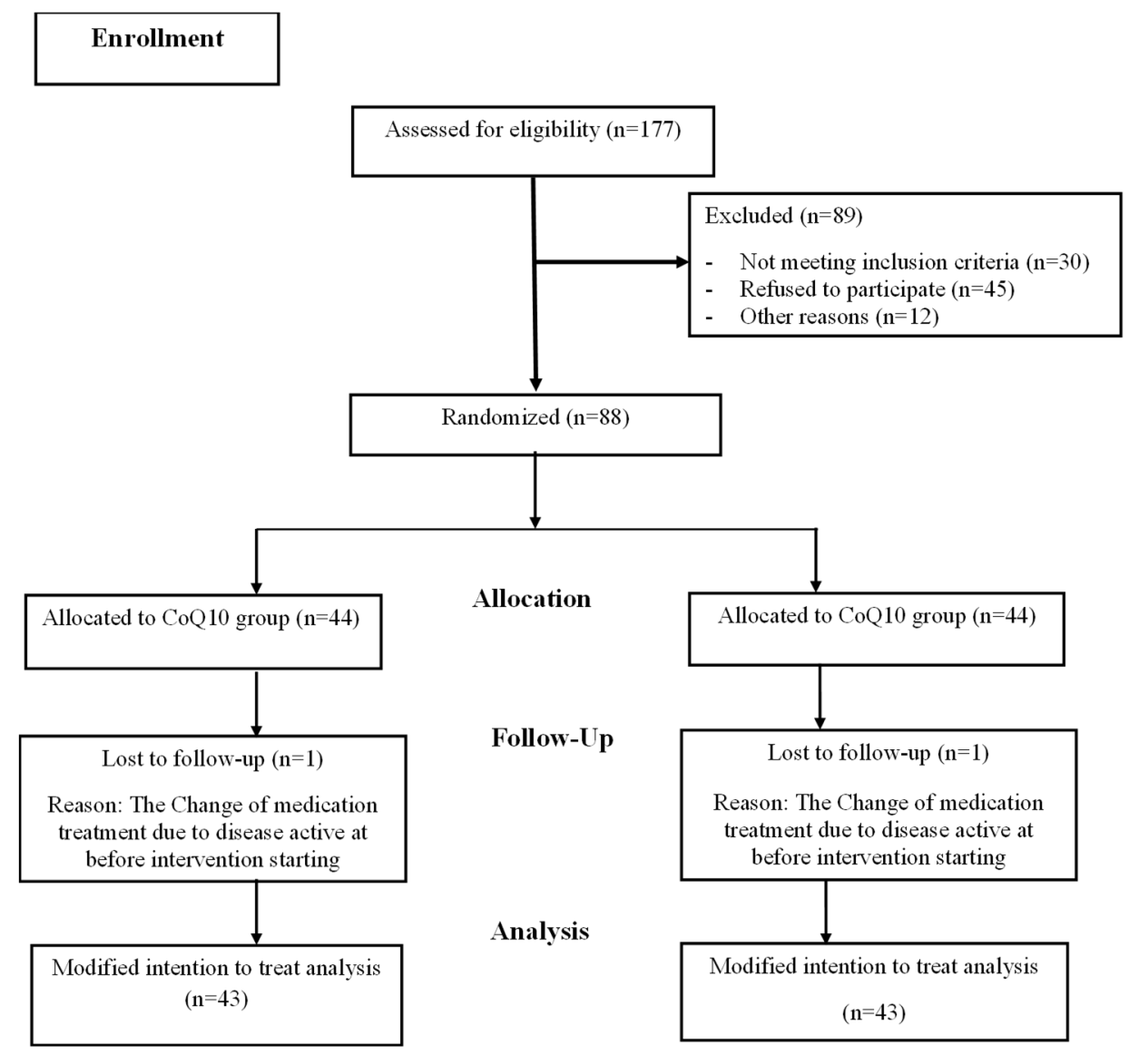

Fig. 1. Patient enrollment flow chart 
week follow-up. Finely, data of 86 eligible patients (43 from the placebo group and 43 from the CoQ10 group) were analyzed. Table 1 outlines the general and demographic features of participants throughout the study.

No significant difference were observed between both groups in terms of baseline height, type of oral medication use, education level, smoking, and marital status. Furthermore, there were no statistically remarkable difference in age (CoQ10 group, 38.39 (8.7) years; placebo group, 40.18 (46) years, respectively, $\mathrm{p}=0.419)$ or gender distribution ( 21 females and 22 males vs. 21 females and 22 males, respectively, $p=1.00$ ) between the two groups. No serious side effects or symptoms were reported due to the consumption of the CoQ10 supplement which was well tolerated by all the patients during the intervention period.

Comparison of participant's dietary intakes: As shown in Table 2 and 3, dietary intakes of calories, total fat, SFA, protein, zinc, selenium, $\mathrm{Cu}$, and some of the antioxidants (vitamin E, A, C, carotenoid) did not significantly differ between both groups during the study; whereas, inter-group comparison illustrated a significant difference in terms of the dietary intake of carbohydrate and PUFA at the onset of the study and the end of the 8 th week. Furthermore, at the baseline of the study the dietary intake of MUFA, total fiber as well as iron significantly differed between both groups $(p=0.012, p=0.016, p=0.001$, respectively).

\section{Changes induced by CoQ10 supplementation}

Intra- and inter- group comparison of anthropometric measures (weight, WC, HC, BMI and WHR) of participants did not display any significant changes in each group (Table 4). As shown in Table 4, after adjustment for dietary intake and baseline value of each variable as covariates, most of the P-values did not change. In contrast, betweengroup analysis indicated significant differences in systolic blood pressure (SBP) and diastolic blood pressure (DBP) at post-intervention stage $(p=0.001$ and $p=0.025$, respectively). Moreover, the changes of SBP and DBP were significant between the two groups $(p<0.001$ and $p<0.001$, respectively). On the other hand, compared to baseline, a significant reduction was seen in SBP and DBP in the CoQ10 group $(p<0.001$ and $p=0.001$, respectively) at the endpoint, but not in the placebo group; indeed, according to the adjusting for the effect of dietary intake and baseline measurements, the significance of the differences in these parameters in the treatment and control groups remained unchanged. While, no statistical significant change was observed in Heart Rate (HR) between both groups 2 months after the intervention $(p=0.925)$; which remained unchanged after adjustment for baseline covariates and dietary intake in both groups.

Compared to the baseline, it has been revealed a statistically remarkable increment in the mean score of IBDQ-32 in the CoQ10 group at the end of the trial, whereas the SCCAIQ mean score reduced markedly $(p=0.001, p<0.001$,

Table 1. Individual characteristics of the participants

\begin{tabular}{|c|c|c|c|}
\hline \multirow[t]{2}{*}{ Variable } & \multicolumn{2}{|c|}{ Group } & \multirow[t]{2}{*}{$\mathrm{p}$} \\
\hline & Placebo $(n=43)$ & CoQ10 $(n=43)$ & \\
\hline Age (years) & $40.18(11.46)$ & $38.39(8.79)$ & $0.419^{*}$ \\
\hline Weight $(\mathrm{Kg})$ & $73.71(11.12)$ & 71.01 (12.99) & $0.304^{*}$ \\
\hline Height $(\mathrm{cm})$ & $170.11(8.86)$ & $168.44(8.48)$ & $0.373^{*}$ \\
\hline $\operatorname{BMI}\left(\mathrm{Kg} / \mathrm{m}^{2}\right)$ & $25.41(2.89)$ & $24.92(3.43)$ & $0.473^{*}$ \\
\hline \multicolumn{4}{|l|}{ Gender } \\
\hline Female & $21(50)$ & $21(50)$ & ${ }^{* *} 1.000$ \\
\hline Male & $22(50)$ & $22(50)$ & \\
\hline \multicolumn{4}{|l|}{ Education Statues } \\
\hline Diploma or lower & $17(53.1)$ & $15(46.9)$ & \\
\hline Academic & $18(54.5)$ & $15(45.5)$ & $0.452^{* *}$ \\
\hline Post-graduate & $8(38.1)$ & $13(61.9)$ & \\
\hline \multicolumn{4}{|l|}{ Marital Statues } \\
\hline Married & $23(50)$ & $23(50)$ & \\
\hline Single & $10(58.8)$ & $7(41.2)$ & ${ }^{* *} 0.220$ \\
\hline Divorced & $0(0)$ & $3(100)$ & \\
\hline \multicolumn{4}{|l|}{ Employment status } \\
\hline Employment & $20(52.6)$ & $18(47.4)$ & ${ }^{* *} 0.664$ \\
\hline Unemployment & $23(47.9)$ & $25(52.1)$ & \\
\hline \multicolumn{4}{|l|}{ Smoking } \\
\hline Yes & $7(77.8)$ & $2(22.2)$ & ${ }^{* *} 0.156$ \\
\hline No & $36(46.8)$ & $41(53.2)$ & \\
\hline \multicolumn{4}{|l|}{ Family history of IBD } \\
\hline Yes & $6(60)$ & $4(40)$ & ${ }^{* *} 0.501$ \\
\hline No & $37(48.7)$ & $39(51.3)$ & \\
\hline \multicolumn{4}{|l|}{ Drug therapy type } \\
\hline Aminosalicylates & $9(47.4)$ & $10(52.6)$ & \\
\hline Aminosalicylates + Immunomodulators & $19(51.4)$ & $18(48.6)$ & \\
\hline Aminosalicylates + Immunomodulators + Asacol' Suppositories & $11(73.3)$ & $4(26.7)$ & ${ }^{* *} 0.122$ \\
\hline Aminosalicylates + Asacol Suppositories + Asacol Enemma & $4(30.8)$ & $9(69.2)$ & \\
\hline Asacol Suppositories + Asacol Enemma & $0(0)$ & $2(100)$ & \\
\hline
\end{tabular}




\begin{tabular}{|c|c|c|c|}
\hline Variable & & Group & $\mathrm{p}$ \\
\hline & Placebo $(n=43)$ & CoQ10 $(n=43)$ & \\
\hline \multicolumn{4}{|l|}{ Energy (Kcal) } \\
\hline Baseline of the study ${ }^{1}$ & $1719.62(379.83)$ & $1717.43(538.20)$ & $0.983^{1}$ \\
\hline End of the study ${ }^{1}$ & $1735.97(402.78)$ & $1744.57(464.44)$ & $0.927^{1}$ \\
\hline Changes & $0(0,0)$ & $0(-212.50,0)$ & $0.036^{1}$ \\
\hline P-Value ${ }^{3}$ & 0.920 & 0.517 & \\
\hline \multicolumn{4}{|l|}{ Protein (gr) } \\
\hline Baseline of the study & $72.93(50.76,89.96)$ & $67.13(58.75,81.70)$ & $0.826^{2}$ \\
\hline End of the study & $71.50(50.19,89.37)$ & $65.60(58.11,80.79)$ & $0.723^{2}$ \\
\hline Changes & $0(0,0)$ & $0(-17.63,0)$ & $0.097^{2}$ \\
\hline P-Value ${ }^{4}$ & 0.328 & 0.381 & \\
\hline \multicolumn{4}{|l|}{ Carbohydrate (gr) } \\
\hline Baseline of the study & $176.85(141.10,231.30)$ & $203(174.2,263.10)$ & $0.017^{2}$ \\
\hline End of the study & $170(148.80,232.60)$ & $202(176.45,269.80)$ & $0.010^{2}$ \\
\hline Changes & $0(0,0)$ & $-2(-36.20,2)$ & $0.141^{2}$ \\
\hline P-Value ${ }^{4}$ & 0.317 & 0.216 & \\
\hline \multicolumn{4}{|l|}{ Total Fat (gr) } \\
\hline Baseline of the study & $54(36.80,67.38)$ & $51.54(38.12,72.95)$ & $0.955^{2}$ \\
\hline End of the study & $50.30(37.25,66.52)$ & $42.73(32.46,70.93)$ & $0.471^{2}$ \\
\hline Changes & $0(-0.10,0.20)$ & $-0.40(-7.56,0)$ & $0.107^{2}$ \\
\hline P-Value ${ }^{4}$ & 0.852 & 0.035 & \\
\hline \multicolumn{4}{|l|}{ PUFA (gr) } \\
\hline Baseline of the study & $8.98(7.34,14.74)$ & $21.30(9.78,31.45)$ & $0.001^{2}$ \\
\hline End of the study & $9.51(6.40,19.42)$ & $21.01(8.01,28.37)$ & $0.009^{2}$ \\
\hline Changes & $0(-0.40,0.14)$ & $0(-0.88,0)$ & 0.735 \\
\hline P-Value ${ }^{4}$ & 0.619 & 0.227 & \\
\hline \multicolumn{4}{|l|}{ MUFA (gr) } \\
\hline Baseline of the study & $13.41(9.21,16.83)$ & $17.44(11.73,23.17)$ & $0.012^{2}$ \\
\hline End of the study & $13.23(7.67,16.49)$ & $15.04(9.7,22.58)$ & $0.054^{2}$ \\
\hline Changes & $0(-0.66,0.78)$ & $0(0,0)$ & 0.600 \\
\hline P-Value ${ }^{4}$ & 0.840 & 0.076 & \\
\hline \multicolumn{4}{|l|}{ SFA (gr) } \\
\hline Baseline of the study & $13.41(9.21,16.83)$ & $17.44(11.73,23.17)$ & $0.174^{2}$ \\
\hline End of the study & $13.23(7.67,16.49)$ & $15.04(9.7,22.58)$ & $0.487^{2}$ \\
\hline Changes & $0(-0.66,0.78)$ & $0(0,0)$ & 0.204 \\
\hline P-Value ${ }^{3}$ & 0.840 & 0.076 & \\
\hline \multicolumn{4}{|l|}{ Total Fiber (gr) } \\
\hline Baseline of the study & $7.17(5.94,9.97)$ & $10.65(7.02,14.69)$ & $0.016^{2}$ \\
\hline End of the study & $9.01(6.28,11.04)$ & $9.4(6.1,14.26)$ & $0.258^{2}$ \\
\hline Changes & $0(0,0.94)$ & $0(-1.62,0)$ & $0.019^{2}$ \\
\hline P-Value ${ }^{4}$ & 0.174 & 0.210 & \\
\hline \multicolumn{4}{|c|}{$\begin{array}{l}\text { Note Data are presented as mean (SD) for Parametric quantitative data and median ( } 25 \text { th and } 75 \text { th percentile) for nonparametric quantitative data. } \\
\mathrm{P} \text { value for between-group comparison of parametric quantitative data using independent-sample t-test. } \\
\mathrm{P} \text { value for between-group comparison of nonparametric quantitative data using Mann-Whitney U-test. } \\
\mathrm{P} \text { value for within-group comparison of nonparametric quantitative data using Wilcoxon signed-rank test. } \\
\mathrm{P} \text { value for within-group comparison of parametric quantitative data using Paired t-test. Abbreviations: PUFA, Polyunsaturated fatty acid; MUFA, Monounsaturated } \\
\text { atty acid; SFA, Saturated fatty acid. }\end{array}$} \\
\hline \multicolumn{4}{|c|}{ Table 3. Between- and within- group comparison of antioxidants intakes in the beginning and the end of the study } \\
\hline Variable & Group & & $\mathrm{p}^{1}$ \\
\hline & Placebo $(n=43)$ & CoQ10 (n=43) & \\
\hline \multicolumn{4}{|l|}{ Vitamin A (mg) } \\
\hline Baseline of the study & $247.35(82.50,413)$ & $278(103.10,543.50)$ & 0.450 \\
\hline End of the study & $257.10(81.10,442.40)$ & $246.95(78.50,448.40)$ & 0.853 \\
\hline Changes & $0(-2,3)$ & $0(-32,3)$ & 0.261 \\
\hline P-Value ${ }^{2}$ & 0.886 & 0.081 & \\
\hline \multicolumn{4}{|l|}{ B-carotene $(\mu \mathrm{g})$} \\
\hline Baseline of the study & $46.09(21.99,96.70)$ & $52.80(26.19,124.20)$ & 0.517 \\
\hline End of the study & $62.50(26.90,118.70)$ & $49.43(28.45,118.55)$ & 0.806 \\
\hline Changes & $0(-4.5,3.6)$ & $0(-0.7,0.01)$ & 0.957 \\
\hline P-Value ${ }^{2}$ & 0.616 & 0.820 & \\
\hline \multicolumn{4}{|l|}{ Vitamin E (mg) } \\
\hline Baseline of the study & $2.19(1.55,3.24)$ & $1.95(1.21,3.39)$ & 0.460 \\
\hline End of the study & $2.30(1.65,3.53)$ & $2.03(1.44,2.24)$ & 0.298 \\
\hline Changes & $0(0,0.13)$ & $0(-0.1,0.32)$ & 0.594 \\
\hline P-Value ${ }^{2}$ & 0.158 & 0.518 & \\
\hline \multicolumn{4}{|l|}{ Vitamin C (mg) } \\
\hline Baseline of the study & $55.10(24.46,88.60)$ & $55.64(33.78,99.50)$ & 0.766 \\
\hline End of the study & $70.36(31.17,100.20)$ & $62.60(32.32,115.05)$ & 0.948 \\
\hline Changes & $0(0,0)$ & $0(0,19.89)$ & 0.212 \\
\hline P-Value ${ }^{2}$ & 0.349 & 0.064 & \\
\hline
\end{tabular}

respectively) but not in the control group. Indeed, comparison of the alterations in these variables from baseline represented that the mean of IBDQ-32 and SCCAIQ scores were statistically different in the CoQ10 group versus the placebo group ( $p<0.002, p<0.003$, respectively). This effect also remained significant after controlling for the baseline 


\begin{tabular}{|c|c|c|c|}
\hline \multirow[t]{2}{*}{ Variable } & \multicolumn{2}{|c|}{ Group } & \multirow[t]{2}{*}{$\mathrm{p}^{1}$} \\
\hline & Placebo $(n=43)$ & CoQ10 $(n=43)$ & \\
\hline \multicolumn{4}{|l|}{ Selenium (mg) } \\
\hline Baseline of the study & $0.07(0.05,0.12)$ & $0.07(0.05,0.10)$ & 0.726 \\
\hline End of the study & $0.06(0.05,0.11)$ & $0.06(0.05,0.09)$ & 0.809 \\
\hline Changes & $0(-0,0)$ & $0(-0.02,0)$ & 0.623 \\
\hline P-Value ${ }^{2}$ & 0.148 & 0.037 & \\
\hline \multicolumn{4}{|l|}{ Zinc (mg) } \\
\hline Baseline of the study & $5.87(5.15,8.07)$ & $6.49(4.79,9.10)$ & 0.678 \\
\hline End of the study & $5.60(4.80,8.10)$ & $6.26(4.51,9.11)$ & 0.526 \\
\hline Changes & $0(-0.1,0.02)$ & $0(-0.99,0.47)$ & 0.599 \\
\hline P-Value ${ }^{2}$ & 0.443 & 0.784 & \\
\hline \multicolumn{4}{|l|}{ Iron (mg) } \\
\hline Baseline of the study & $10.67(8.33,13.76)$ & $10.33(7.16,14.04)$ & 0.001 \\
\hline End of the study & $12.54(9.63,15.62)$ & $10.60(7.63,15.63)$ & 0.121 \\
\hline Changes & $0(2.37,0)$ & $0(0,0.28)$ & 0.002 \\
\hline P-Value ${ }^{2}$ & 0.049 & 0.196 & \\
\hline \multicolumn{4}{|l|}{$\mathrm{Cu}(\mathrm{mg})$} \\
\hline Baseline of the study & $0.76(0.59,1.25)$ & $0.91(0.64,1.24)$ & 0.595 \\
\hline End of the study & $0.90(0.60,1.43)$ & $0.92(0.53,1.33)$ & 0.887 \\
\hline Changes & $0(-0,0)$ & $0(-0.09,0.11)$ & 0.897 \\
\hline P-Value ${ }^{2}$ & 0.313 & 0.772 & \\
\hline
\end{tabular}

Note Nonparametric quantitative data are presented as median (25th and 75 th percentile).

${ }^{1} \mathrm{P}$ value for between-group comparison of nonparametric quantitative data using Mann-Whitney U-test.

${ }^{2} \mathrm{P}$ value for within-group comparison of nonparametric quantitative data using Wilcoxon signed-rank test.

Table 4. Between- and within- group comparison of the metabolic parameters in the beginning and the end of the study

\begin{tabular}{|c|c|c|c|c|c|}
\hline \multirow[t]{2}{*}{ Variable } & \multicolumn{2}{|c|}{ Group } & \multirow[t]{2}{*}{$\mathrm{P}^{2}$} & \multirow[t]{2}{*}{ P-Adjusted $^{3}$} & \multirow[t]{2}{*}{ P-Adjusted $^{4}$} \\
\hline & Placebo $(n=43)$ & CoQ10 $(n=43)$ & & & \\
\hline \multicolumn{6}{|l|}{ Weight (Kg) } \\
\hline Baseline of the study & $73.71(11.12)$ & $71.01(12.99)$ & 0.304 & & \\
\hline End of the study & $73.67(11.09)$ & $71.01(12.65)$ & 0.302 & 0.936 & 0.358 \\
\hline Changes & $-0.10(-0.55,0.32)$ & $0(-0.60,0.70)$ & 0.619 & & \\
\hline P-Value ${ }^{4}$ & 0.504 & 0.833 & & & \\
\hline \multicolumn{6}{|l|}{ BMI $\left(\mathrm{Kg} / \mathrm{m}^{2}\right)$} \\
\hline Baseline of the study & $25.41(2.89)$ & $24.92(3.43)$ & 0.473 & & \\
\hline End of the study & $25.39(2.88)$ & $24.92(3.32)$ & 0.484 & 0.942 & 0.474 \\
\hline Changes & $0.03(-0.16,0.10)-$ & $0(-0.17,0.26)$ & 0.601 & & \\
\hline P-Value ${ }^{4}$ & 0.438 & 0.850 & & & \\
\hline \multicolumn{6}{|l|}{$\mathrm{WC}(\mathrm{cm})$} \\
\hline Baseline of the study & $93.58(12.16)$ & $91.51(11.38)$ & 0.417 & & \\
\hline End of the study & $93.98(12.78)$ & $91.77(10.30)$ & 0.380 & 0.724 & 0.581 \\
\hline Changes & $0(-0.10,0.10)$ & $0(-0.5,1)$ & 0.783 & & \\
\hline P-Valu ${ }^{4}$ & 0.782 & 0.646 & & & \\
\hline \multicolumn{6}{|l|}{$\mathrm{HC}$} \\
\hline Baseline of the study & $105.44(7.01)$ & $103.20(7.82)$ & 0.167 & & \\
\hline End of the study & $105.29(7.05)$ & $103.23(7.33)$ & 0.188 & 0.973 & 0.818 \\
\hline Changes & $0(0,0)$ & $0(-1,1)$ & 0.674 & & \\
\hline P-Value ${ }^{4}$ & 0.913 & 0.934 & & & \\
\hline
\end{tabular}

values of each variable as covariates and dietary intake (Table 5). Concerning the mean score of IPAQ, no significant within- and between-group differences in physical activity levels were seen in both groups at pre- and post-intervention stages (Table 5).

\section{Discussion}

Up to the best of the author's knowledge, the current research is the randomized, double-blind, placebo-controlled trial which assessed the efficacy of CoQ10 supplementation on the severity of clinical disease activity and quality of life in adult patients with mild-to-moderate remission UC for the first time. The findings of the our study highlight a statistically significant decline in the clinical activity index score (assessed by the SCCAIQ) and a substantial the QOL improvement (assessed by the IBDQ-32 scores) in UC patients after $200 \mathrm{mg}$ CoQ10 supplementation for 8 weeks. Based on existing data, several clinical studies regarding CoQ10 or supplements containing CoQ10 and QOL with contradictory results have been carried out using different scales for QOL $(15,20,21,24,26-28)$. Our findings are in line with a recent trial revealing that CoQ10 (150 mg/day) to be superior to placebo for improving and increasing QOL for up to 12 months in 41 patients with functional gastrointestinal disorders in Japan (26). Besides, another recent study reported the beneficial effect of commonly-used dosage of ubiquinol intake (100 mg/day) after an 8-week inter- 


\begin{tabular}{|c|c|c|c|c|c|}
\hline \multirow[t]{2}{*}{ Variable } & \multicolumn{2}{|c|}{ Group } & \multirow[t]{2}{*}{$\mathrm{P}$-value $^{1}$} & \multirow[t]{2}{*}{ P-Adjusted $^{2}$} & \multirow[t]{2}{*}{ P-Adjusted $^{3}$} \\
\hline & Placebo $(n=43)$ & CoQ10 (n=43) & & & \\
\hline \multicolumn{6}{|l|}{ WHR } \\
\hline Baseline of the study & $0.88(0.08)$ & $0.88(0.06)$ & 0.922 & & \\
\hline End of the study & $0.89(0.09)$ & $0.88(0.05)$ & 0.826 & 0.744 & 0.458 \\
\hline Changes & $0(0,0)$ & $0(0,0)$ & 0.990 & & \\
\hline P-Value $^{4}$ & 0.694 & 0.732 & & & \\
\hline \multicolumn{6}{|l|}{ SBP (mmHg) } \\
\hline Baseline of the study & $125.23(12.73)$ & $121.97(12.10)$ & 0.228 & & \\
\hline End of the study & $125.83(12.13)$ & $117.09(12.26)$ & 0.001 & $<0.001$ & 0.001 \\
\hline Changes & $0.5(-1,1)$ & $-3(-7,-2)$ & $0.001<$ & & \\
\hline $\mathrm{P}^{-V^{2}}$ & 0.091 & $0.001<$ & & & \\
\hline \multicolumn{6}{|l|}{ DBP (mmHg) } \\
\hline Baseline of the study & $80.23(11.62)$ & $78.40(8.43)$ & 0.406 & & \\
\hline End of the study & $80.66(11.18)$ & $75.96(7.61)$ & 0.025 & 0.003 & 0.002 \\
\hline Changes & $0(-1,1)$ & $-2(-5,0)$ & $0.001<$ & & \\
\hline P-Value ${ }^{4}$ & 0.417 & 0.001 & & & \\
\hline \multicolumn{6}{|l|}{ HR } \\
\hline Baseline of the study & $76.55(11.03)$ & $77.95(9.97)$ & 0.540 & & \\
\hline End of the study & $76.10(11.17)$ & $78.15(9.08)$ & 0.335 & 0.444 & 0.289 \\
\hline Changes & $0(-1,0)$ & $0(-1,1)$ & 0.925 & & \\
\hline P-Value $^{4}$ & 0.424 & 0.984 & & & \\
\hline
\end{tabular}

Table 5. Between- and within- group comparison of the disease severity and quality of life and physical activity in the beginning and the end of the study

\begin{tabular}{|c|c|c|c|c|c|}
\hline \multirow[t]{2}{*}{ Variable } & \multicolumn{2}{|c|}{ Group } & \multirow[t]{2}{*}{ P-value $^{1}$} & \multirow[t]{2}{*}{ P-Adjusted ${ }^{2}$} & \multirow[t]{2}{*}{ P-Adjusted $^{3}$} \\
\hline & Placebo $(n=43)$ & CoQ10 $(n=43)$ & & & \\
\hline \multicolumn{6}{|l|}{ SCCAI score } \\
\hline Baseline of the study & $3.06(0.88)$ & $3.06(0.88)$ & 1.000 & & \\
\hline End of the study & $3.04(1.11)$ & $2.13(0.96)$ & $0.001<$ & $<0.001$ & 0.001 \\
\hline Changes & $-0.02(1.51)$ & $-0.93(1.22)$ & 0.003 & & \\
\hline P-Value ${ }^{4}$ & 0.921 & $0.001<$ & & & \\
\hline \multicolumn{6}{|l|}{ IBDQ-32 } \\
\hline Baseline of the study & $162.48(8.94)$ & $162(8.86)$ & 0.800 & & \\
\hline End of the study & $159.51(13.43)$ & $169.39(9.33)$ & $<0.001$ & $<0.001$ & 0.001 \\
\hline Changes & $-2.90(0.86)$ & $7.39(13.58)$ & 0.002 & & \\
\hline P-Value ${ }^{4}$ & 0.257 & 0.001 & & & \\
\hline \multicolumn{6}{|l|}{ PAL (MET-hr/week) } \\
\hline Baseline of the study & $36.59(3.29)$ & $36.54(3.40)$ & 0.947 & & \\
\hline End of the study & $36.75(3.20)$ & $36.67(3.22)$ & 0.903 & 0.868 & 0.717 \\
\hline Changes & $0.165(1.18)$ & $0.12(1.23)$ & 0.885 & & \\
\hline P-Value ${ }^{4}$ & 0.364 & 0.500 & & & \\
\hline
\end{tabular}

Note Data are presented as mean (SD) for parametric quantitative data

${ }^{1} \mathrm{P}$ value for between-group comparison of parametric quantitative data using independent-sample t-test.

${ }^{2} \mathrm{P}$ value using ANCOVA test adjusted for baseline measures.

${ }_{3}^{3}$ Bootstrap p-values reported regarding non-normal distribution of dependent variable, treatment type, carbohydrate, iron and fiber.

${ }^{4} \mathrm{P}$ value for within-group comparison of parametric quantitative data using Paired t-test.

Abbreviations: IBDQ-32, Inflammatory Bowel Disease Questionnaire -32; SCCAI, Simple Colitis Clinical Activity Index. PAL, Physical activity level.

vention in the QOL of breast cancer patients in Iranian undergoing tamoxifen therapy (29). However, in contrast with prior trials, Lesser et al. did not find any indication of a CoQ10 efficacy on QOL in newly diagnosed patients with breast cancer following the supplementation $300 \mathrm{mg}$ after 24 weeks of treatment in USA (28). According to the literature search, no clinical trial has reported the effects of CoQ10 on disease activity in UC patients. The obtained results of the current study in consistence with previous investigations in the experimental models, which have found a protective effect of CoQ10 (10 or 30 and $100 \mathrm{mg} / \mathrm{kg}$, orally for 8 days) in a dose-related response on clinical evaluation and macroscopic scoring of $\operatorname{UC}(22,23)$. Based on the existing evidence, it has been revealed the underline how the oral intake of CoQ10 improves QOL (33). One possible mechanism could be attributed in part to wellacknowledged bioenergetics role of CoQ10 that plays as an essential cofactor in the mitochondrial respiratory chain (ETC) that is implicated in aerobic respiration for cellular production of ATP $(28,33)$. Moreover, CoQ10, as a potent lipophilic antioxidant could be neutralized harmful free radical species in lipid and mitochondrial membranes and regenerated several antioxidants $(33,34)$. A part of the beneficial effects observed by CoQ10 might be related to its anti-inflammatory properties on the immune-inflammatory cytokines such as tumor necrosis factor-alpha and inteleukin-6 via down-regulating nuclear factor NF- $\kappa B-d e-$ pendent gene expression and -activation by the ROS according to the present and previous studies $(35,36)$. Furthermore, it should be mentioned that both oxidative stress and excessive expression of inflammatory cytokines as major causes have involved in the pathogenic pathway of UC which, in turn, encouraged the use of antioxidant compounds with anti-inflammatory properties (22). Indeed, it has been proposed that CoQ10 may be applicable in the treatment of numerous medical conditions in which inflammation, oxidative and nitrosative stress play a part by improving exercise tolerance, reducing muscle weakness and 
creating objective improvements in exercise parameters, viz., restoring oxygen consumption and elevating walking distance (37).

As evidenced in this study in the intervention group, the clinical activity of UC patients along with the QOL markedly was also improved by CoQ10. Reports, in this regard, have demonstrated that IBD severity can be considered as the main predictor of both physical and mental HR-QOL, because IBD has negative effects on an individual's healthrelated quality of life (38). Another possible mechanism that might be involved in of lowering disease severity by CoQ10 supplementation, could be due to improving the electron flow in ETC; which could ultimately contribute to further lower ROS production and subsequently reducing inflammatory pathways $(22,23)$.

At other extremes, several other studies similar to the present study have used the SCCAI to assess disease severity in UC subjects $(8,30)$, which was recently considered to be more reflective of actual disease activity in comparison to other clinical scores (39). Although it should be noted that serum CoQ10 levels may be influenced by a variety of factors (e.g., age, race, plasma lipid levels, and use of concurrent medications) $(9,28)$.

Moreover, the findings of our study represented a significant reduction in SBP and DBP in subjects with UC following CoQ10 supplementation for 8 weeks; but this effect was not seen for heart rate in both groups. This is in accordance with the other reports, which suggested blood pressure-lowering efficacy of CoQ10 (40-43). This suggests an inverse correlation between serum CoQ10 levels and blood pressure (42). However, these findings contrast with previous human studies and meta-analysis (44-46). It seems that this controversy, maybe because of the time frame of the study, different pathophysiology, baseline characteristics, baseline CoQ10 concentrations, and CoQ10 dosing regimens. Hence, the findings of CoQ10 impact on blood pressure control should be interpreted cautiously. Noteworthy is that to date, the antihypertensive mechanism of action of CoQ10 is still unknown $(40,47)$. Based on the evidence, the CoQ10-mediated reduction of blood pressure observed in the current work may be linked to its direct effects on vascular endothelium and smooth muscle through the ability to counteract vasoconstriction $(40,42,47)$. Besides its well documented antioxidant characters, CoQ10 might have also indirect effects on vascular function by amplifying the NO availability rate and prevention of the inactivation of endothelial nitric oxide (NO) in response to superoxide radicals which may result from quenching free radicals such as superoxide by CoQ10 $(40,42)$. Additionally, it has been suggested that CoQ10 can lead to enhancing prostacyclin production and the sensitivity of arterial smooth muscles to prostacyclin (42). Studies have also proven the inverse relationship between serum CoQ10 levels with inflammatory cytokines and vascular endothelial biomarkers which could be reversed by CoQ10 supplementation; consequently, both systolic/diastolic blood pressures can diminish following the rising plasma CoQ10 levels (42).

In the current investigation, we failed to detect significant changes of the anthropometric indices following 8 weeks of CoQ10 consumption. This finding appears to be reasonable because that we did not impose a restriction of energy intake or increasing physical activity level of study participants; considering that there was not seen any significant changes in calorie intake and physical activity status in the intervention and control groups at the onset and the endpoint of the study. Similar to our results, the meta-analysis of seventeen RCTs recently demonstrated that CoQ10 supplementation has no beneficial effect on body weight and BMI of patients (48). The same results were found in several other previous studies, which have reported that oral CoQ10 intake (100 or $200 \mathrm{mg} /$ day) has not influenced anthropometric parameters of patients in clinical studies for 12- or 8-week intervention $(41,43,46,49)$. In contrast to the data presented here, Abbasalizad Farhangi et al. found that an oral CoQ10 administration in NAFLD patients decreased several anthropometric variables after 4 weeks (50). According to Hosseinzadeh-Attar, who observed a remarkable decrease in weight, BMI and WC of diabetic type 2 patients by CoQ10 supplement therapy (51). Different populations of these studies is a probable reason for this inconsistency. A possible mechanism of CoQ10 action on weight management may be associated with its anti-adipogenic characters which in turn inhibits adipocyte differentiation through AMPK-mediated PPAR $\alpha$ pathway (48). On the other hand, CoQ10 seems to increase energy expenditure and fat oxidation by down-regulating the gene expression of enzyme fatty acid synthesis and endogenous lipid synthesis via induction protein gene expression-related to energy expenditure (48).

The current investigation has several strengths, including study size, homogeneity of the study population, a high compliance of the participants, a low drop-out rate, successful blinding, it's double-blind, placebo-controlled design, and strict exclusion criteria, which reduced the potential effects of confounding factors. Additionally, this is the first research about the effect of CoQ10 in UC patients, to our knowledge. Nonetheless, one of the main weak aspects of this trial was related to the absence of measurement of CoQ10 serum levels throughout the study. A further limitation was due to the fact that the disease activity of UC patients was not evaluated by colonoscopy because of their unwillingness to perform this procedure during the intervention period. Although, it has recently been demonstrated that SCCAIQ has a strong relationship with colonoscopy and other clinical evaluations in UC patients else; therefore, our results need to be interpreted with caution.

\section{Conclusion}

In conclusion, the findings of this clinical trial revealed that $200 \mathrm{mg} /$ day CoQ10 supplementation might markedly boost the quality of life and decrement severity of disease in patients with mild-to-moderate UC. Additionally, the results showed a beneficial effect of CoQ10 on blood pressure control in participants, while, its administration could not affect any anthropometric parameters following intervention period. Further clinical trials for investigating the exact mechanism of CoQ10 in inflammatory bowel disease are necessary. 


\section{Acknowledgement}

The authors wish to thank all participants, Rasoul-eAkram Hospital Colorectal Research Center, Iran University of Medical Sciences. This paper was adapted from a $\mathrm{PhD}$ dissertation, which was supported by Research ViceChancellor, Iran University of Medical Sciences, Tehran, Iran (No: IR.IUMS.REC 96-02-27.31412).

\section{Conflict of Interests}

The authors declare that they have no competing interests.

\section{References}

1. Seyedian SS, Nokhostin F, Malamir MD. A review of the diagnosis, prevention, and treatment methods of inflammatory bowel disease. J Med Life. 2019;12(2):113.

2. Tanaka M, Kazuma K. Ulcerative colitis: factors affecting difficulties of life and psychological well being of patients in remission. J Clin Nurs. 2005;14(1):65-73.

3. Kuriyama M, Kato J, Kuwaki K, Morimoto N, Nawa T, Fujimoto T, et al. Japan West Ulcerative Colitis Study Group: Clinical factors that impair health- related quality of life in ulcerative colitis patients vary with the disease duration. Eur J Gastroenterol Hepatol. 2008;20:63441.

4. Ng SC, Shi HY, Hamidi N, Underwood FE, Tang W, Benchimol EI, et al. Worldwide incidence and prevalence of inflammatory bowel disease in the 21st century: a systematic review of population-based studies. Lancet. 2017;390(10114):2769-78.

5. Kamm MA. Rapid changes in epidemiology of inflammatory bowel disease. Lancet. 2018;390(10114):2741-2.

6. Hosseini RS, Mansour-Ghanaei F, Shafaghi A, Hojati A, Joukar F, Roushan ZA, et al. Exacerbation causes among inflammatory bowel disease patients in Guilan Province north of Iran. JCOL. 2019;39(2):138-44.

7. Malekzadeh MM, Vahedi H, Gohari K, Mehdipour P, Sepanlou SG, Ebrahimi Daryani N, et al. Emerging epidemic of inflammatory bowel disease in a middle income country: a nation-wide study from Iran. Arch Iran Med. 2016:1-14.

8. Samsami-kor M, Daryani NE, Asl PR, Hekmatdoost A. Antiinflammatory effects of resveratrol in patients with ulcerative colitis: a randomized, double-blind, placebo-controlled pilot study. Arch Med Res. 2015;46(4):280-5.

9. Christiansen LK, Lo B, Bendtsen F, Vind I, Vester-Andersen MK, Burisch J. Health-related quality of life in inflammatory bowel disease in a Danish population-based inception cohort. United European Gastroenterol J. 2019;7(7):942-54.

10. Tsoukka M, Jelastopulu E, Lavranos G, Charalambous G. Estimation of quality of life in Cypriot patients with inflammatory bowel disease. World J Gastroenterol. 2017;23(1):121.

11. Kalafateli M, Triantos C, Theocharis G, Giannakopoulou D, Koutroumpakis E, Chronis A, et al. Health-related quality of life in patients with inflammatory bowel disease: a single-center experience. Ann Gastroenterol. 2013;26(3):243.

12. Armuzzi A, Tarallo M, Lucas J, Bluff D, Hoskin B, Bargo D, et al. The association between disease activity and patient-reported outcomes in patients with moderate-to-severe ulcerative colitis in the United States and Europe. BMC Gastroenterol. 2020;20(1):18.

13. Hagelund LM, Elkjær Stallknecht S, Jensen HH. Quality of life and patient preferences among Danish patients with ulcerative colitisresults from a survey study. Curr Med Res Opin. 2020;36(5):771-9.

14. Haapamäki J, Turunen U, Roine RP, Färkkilä MA, Arkkila PE. Impact of demographic factors, medication and symptoms on disease-specific quality of life in inflammatory bowel disease. Qual Life Res. 2009;18(8):961-9.

15. Sharifi MH, Eftekhari MH, Ostovan MA, Rezaianazadeh A. Effects of a therapeutic lifestyle change diet and supplementation with Q10 plus L-carnitine on quality of life in patients with myocardial infarction: a randomized clinical trial. J Cardiovasc Thorac Res. 2017;9(1):21.

16. Sainsbury A, Heatley R. Psychosocial factors in the quality of life of patients with inflammatory bowel disease. Aliment Pharmacol Ther. 2005;21(5):499-508.
17. Park S-K, Ko BM, Goong HJ, Seo JY, Lee SH, Baek HL, et al. Short health scale: A valid measure of health-related quality of life in Koreanspeaking patients with inflammatory bowel disease. World J Gastroenterol. 2017;23(19):3530.

18. Bijari B, Soltani B. Validation of the Persian Version of Inflammatory Bowel Disease Questionnaire in Patients Who Referred to Clinics and Hospitals of Birjand University of Medical Sciences, Iran. Curr Colorectal Cancer Rep. 2017;5(1-2).

19. Dave M, Loftus Jr EV. Mucosal healing in inflammatory bowel disease-a true paradigm of success?. Gastroenterol Hepatol 2012;8(1):29.

20. Johansson P, Dahlström Ö, Dahlström U, Alehagen U. Improved health-related quality of life, and more days out of hospital with supplementation with selenium and coenzyme Q10 combined. Results from a double blind, placebo-controlled prospective study. Aging Ment Health. 2015;19(9):870-7.

21. Kinoshita T, Maruyama K, Tanigawa T. The effects of long-term Ubiquinol intake on improving the Quality of Life of community residents. Funct Food Health Dis. 2016;6(1):16-32.

22. Khodir AE, Atef H, Said E, ElKashef HA, Salem HA. Implication of Nrf2/HO-1 pathway in the coloprotective effect of coenzyme Q10 against experimentally induced ulcerative colitis. Inflammopharmacology. 2017;25(1):119-35.

23. El Morsy EM, Kamel R, Ahmed MA. Attenuating effects of coenzyme Q10 and amlodipine in ulcerative colitis model in rats. Immunopharmacol Immunotoxicol. 2015;37(3):244-51.

24. FG Cicero A, Colletti A. Nutraceuticals and dietary supplements to improve quality of life and outcomes in heart failure patients. Curr Pharm Des. 2017;23(8):1265-72.

25. Mehrabani S, Arab A. Effect of coenzyme Q10 supplementation on fatigue: A systematic review of interventional studies. Complement Ther. Med. 2019;43:181-187.

26. Suzuki S, Gotoda T, Kusano C, Ikehara H, Miyakoshi Y, Fujii K. Effect of Ubiquinol Intake on Defecation Frequency and Stool Form: A Prospective, Double-Blinded, Randomized Control Study. J Med Food. 2019;22(1):81-6.

27. Marinari S, Manigrasso MR, De Benedetto F. Effects of nutraceutical diet integration, with coenzyme Q 10 (Q-Ter multicomposite) and creatine, on dyspnea, exercise tolerance, and quality of life in COPD patients with chronic respiratory failure. Multidiscip Respir Med. 2013;8(1):40.

28. Lesser GJ, Case D, Stark N, Williford S, Giguere J, Garino LA, et al. A Randomized double-blind, placebo-controlled study of oral coenzyme Q10 to relieve self-reported treatment related fatigue in newly diagnosed patients with breast cancer. J Support Oncol. 2013;11(1):31.

29. Hosseini SA, Zahrooni N, Ahmadzadeh A, Ahmadiangali K, Assarehzadegan M-A. The Effect of CoQ(10) Supplementation on Quality of Life in Women with Breast Cancer Undergoing Tamoxifen Therapy: A Double-Blind, Placebo-Controlled, Randomized Clinical Trial. Psychol Res Behav Manag. 2020;13:151-9.

30. Karimi S, Tabataba-vakili S, Yari Z, Alborzi F, Hedayati M, Ebrahimi-Daryani N, et al. The effects of two vitamin D regimens on ulcerative colitis activity index, quality of life and oxidant/anti-oxidant status. Nutr J. 2019;18(1):16.

31. Vasheghani-Farahani A, Tahmasbi M, Asheri H, Ashraf H, Nedjat S, Kordi R. The Persian, last 7-day, long form of the International Physical Activity Questionnaire: translation and validation study. Asian J Sports Med. 2011;2(2):106-16.

32. Sadeghisani M, Dehghan Manshadi F, Azimi H, Montazeri A. Validity and Reliability of the Persian Version of Baecke Habitual Physical Activity Questionnaire in Healthy Subjects. Asian J Sports Med. 2016;7(3):e31778.

33. Motohashi N, Gallagher R, Anuradha V, Gollapudi R. Co-enzyme Q10 (Ubiquinone): It's Implication in Improving the Life Style of the Elderly. Med Clin Rev. 2017;3(10).

34. Brauner H, Lüthje P, Grünler J, Ekberg N, Dallner G, Brismar K, et al. Markers of innate immune activity in patients with type 1 and type 2 diabetes mellitus and the effect of the anti-oxidant coenzyme Q 10 on inflammatory activity. Clin Exp Immunol. 2014;177(2):478-82.

35. Farsi F, Heshmati J, Janani L, Irandoost $\mathrm{P}$, Mesri Alamdari N, Keshtkar A, et al. Can coenzyme Q10 supplementation effectively reduce human tumour necrosis factor- $\alpha$ and interleukin- 6 levels in chronic diseases? Protocol for a systematic review and meta-analysis of randomised controlled trials. BMJ Open. 2017;7(10):e016841-e. 
36. Farsi F, Heshmati J, Keshtkar A, Irandoost P, Alamdari NM, Akbari A, et al. Can coenzyme Q10 supplementation effectively reduce human tumor necrosis factor- $\alpha$ and interleukin- 6 levels in chronic inflammatory diseases? A systematic review and meta-analysis of randomized controlled trials. Pharmacol Res. 2019;148:104290.

37. Morris G, Anderson G, Berk M, Maes M. Coenzyme Q10 depletion in medical and neuropsychiatric disorders: potential repercussions and therapeutic implications. Cell Mol Neurobiol. 2013;48(3):883-903.

38. Pizzi LT, Weston CM, Goldfarb NI, Moretti D, Cobb N, Howell JB, et al. Impact of chronic conditions on quality of life in patients with inflammatory bowel disease. Inflamm Bowel Dis. 2006;12(1):47-52.

39. Lang A, Salomon N, Wu JC, Kopylov U, Lahat A, Har-Noy O, et al. Curcumin in combination with mesalamine induces remission in patients with mild-to-moderate ulcerative colitis in a randomized controlled trial. Clin Gastroenterol Hepatol. 2015;13(8):1444-9. e1

40. Tabrizi R, Akbari M, Sharifi N, Lankarani KB, Moosazadeh M, Kolahdooz F, et al. The effects of coenzyme Q10 supplementation on blood pressures among patients with metabolic diseases: a systematic review and meta-analysis of randomized controlled trials. High Blood Press Cardiovasc Prev. 2018;25(1):41-50.

41. Ho MJ, Li EC, Wright JM. Blood pressure lowering efficacy of coenzyme Q10 for primary hypertension. Cochrane Database Syst Rev. 2016;3.

42. Mohseni M, Vafa MR, Hajimiresmail SJ, Zarrati M, Forushani AR, Bitarafan V, et al. Effects of coenzyme q10 supplementation on serum lipoproteins, plasma fibrinogen, and blood pressure in patients with hyperlipidemia and myocardial infarction. Iran Red Crescent Med J. 2014;16(10):e16433.

43. Rosenfeldt FL, Haas SJ, Krum H, Hadj A, Ng K, Leong JY, et al. Coenzyme Q 10 in the treatment of hypertension: a meta-analysis of the clinical trials. J Hum Hypertens. 2007;21(4):297.

44. Ho MJ, Bellusci A, Wright JM. Blood pressure lowering efficacy of coenzyme Q10 for primary hypertension. Cochrane Database Syst Rev. 2009(4):1-3.

45. Young JM, Florkowski CM, Molyneux SL, McEwan RG, Frampton $\mathrm{CM}$, Nicholls MG, et al. A randomized, double-blind, placebocontrolled crossover study of coenzyme Q10 therapy in hypertensive patients with the metabolic syndrome. Am J Hypertens. 2012;25(2):261-70.

46. Abdollahzad H, Alipour B, Aghdashi MA, Jafarabadi MA. Coenzyme Q10 supplementation in patients with rheumatoid arthritis: Are there any effects on cardiovascular risk factors? J Integr Med. 2015;7(5):534-9.

47. Ayers J, Cook J, Koenig RA, Sisson EM, Dixon DL. Recent developments in the role of Coenzyme Q10 for coronary heart disease: a systematic review. Curr Atheroscler Rep. 2018;20(6):29.

48. Saboori S, Rad EY, Mardani M, Khosroshahi MZ, Nouri Y, Falahi E. Effect of Q10 supplementation on body weight and body mass index: A systematic review and meta-analysis of randomized controlled clinical trials. Diabetes Metab Syndr. 2019;13:1179-1185.

49. Raygan F, Rezavandi Z, Tehrani SD, Farrokhian A, Asemi Z. The effects of coenzyme Q10 administration on glucose homeostasis parameters, lipid profiles, biomarkers of inflammation and oxidative stress in patients with metabolic syndrome. Eur J Nutr. 2016;55(8):2357-64.

50. Farhangi MA, Alipour B, Jafarvand E, Khoshbaten M. Oral coenzyme Q10 supplementation in patients with nonalcoholic fatty liver disease: effects on serum vaspin, chemerin, pentraxin 3, insulin resistance and oxidative stress. Arch Med Res. 2014;45(7):589-95.

51. Hosseinzadeh-Attar M, Kolahdouz Mohammadi R, Eshraghian M, Nakhjavani M, Khorrami E, Ebadi M, et al. Reduction in asymmetric dimethylarginine plasma levels by coenzyme Q10 supplementation in patients with type 2 diabetes mellitus. Minerva Endocrinol. 2015;40(4):259-66. 